\title{
3 Research Square \\ Reinforcing the Supply Chain of Umifenovir and Other Antiviral Drugs with Retrosynthetic Software
}

Yingfu Lin

University of Michigan

\section{Zirong Zhang}

University of Michigan

\section{Babak Mahjour}

University of Michigan

Di Wang

University of Michigan

Rui Zhang

University of Michigan

Eunjae Shim

University of Michigan

Andrew McGrath

University of Michigan

Yuning Shen

University of Michigan

\section{Nadia Brugger}

MilliporeSigma

Rachel Turnbull

MilliporeSigma

Sarah Trice

MilliporeSigma

Shashi Jasty

MilliporeSigma

Tim Cernak ( $\nabla$ tcernak@med.umich.edu )

University of Michigan https://orcid.org/0000-0001-5407-0643

\section{Article}

Keywords: supply chain, umifenovir, antiviral drugs, retrosynthetic software

Posted Date: August 19th, 2021 
DOl: https://doi.org/10.21203/rs.3.rs-820369/v1

License: (c) (1) This work is licensed under a Creative Commons Attribution 4.0 International License. Read Full License

Version of Record: A version of this preprint was published at Nature Communications on December 1st, 2021. See the published version at https://doi.org/10.1038/s41467-021-27547-3. 


\section{Abstract}

The global disruption caused by the 2020 coronavirus pandemic stressed the supply chain of many products, including pharmaceuticals. Multiple drug repurposing studies for COVID-19 are now underway. If a winning therapeutic emerges, it is unlikely that the existing inventory of the medicine, or even the chemical raw materials needed to synthesize it, will be available in the quantities required. We used retrosynthetic software to arrive at alternate chemical supply chains for the antiviral drug umifenovir, as well as eleven other antiviral and anti-inflammatory drugs. We have experimentally validated four routes to umifenovir and one route to bromhexine. In several instances, the software utilizes $\mathrm{C}-\mathrm{H}$ functionalization logic, and one route to umifenovir employs functionalization of six $\mathrm{C}-\mathrm{H}$ bonds. The general strategy we apply can be used to identify distinct starting materials, and relieve stress on existing supply chains.

\section{Introduction}

In 2020, the scourge of coronavirus highlighted the fragility of diverse supply chains, affecting the world's pipeline of hand sanitizer (1), toilet paper (2), and pharmaceutical starting materials (3). A diverse array of antiviral and anti-inflammatory drugs was investigated in the hopes that an existing medicine could be repurposed for use against COVID-19 (4, 5). The scale of the pandemic (6) would easily stress the pharmaceutical supply chain (7). For instance, at the time of our study, the only drug with an emergency use authorization for treating COVID-19 was remdesivir (1). While its producer, Gilead Sciences, Inc., ramped up production of $\mathbf{1}$ significantly, there were only 5,000 doses of 1 available when the outbreak began (8).

We realized that the availability of alternative starting materials to promising synthetic therapeutics could alleviate pressure on supply chains. We reasoned that modern retrosynthetic software could be used to analyze the diverse chemical agents that were being studied clinically and pre-clinically when we initiated our study (4). For the sake of preparedness, we chose to consider multiple therapeutic synthetic targets simultaneously, even though many would eventually prove to be irrelevant in the war on COVID-19. We focused our experimental attention on umifenovir (2), which had been used against SARS-CoV1, and is effective against SARS-CoV2 in vitro (9). Automated retrosynthesis has already been used to design contingency plans for the investigational COVID-19 therapeutics hydroxychloroquine and remdesivir (1) (10). This reaction-centric study identified novel sequences computationally, but the routes initiate with known starting materials, or from starting materials that added significantly to route length or reagent cost. Our study focusses specifically on a starting material-centric analysis to identify distinct raw materials that are of comparable cost, and which feature in routes of comparable length to the known routes. We also sought to simultaneously explore diverse targets. From our perspective, it was not detrimental to intercept established synthetic routes to the selected drugs, as long as the overall route had a competitive step-count and initiated with distinct starting materials of comparable or better price. The simultaneous design of multistep preparative routes to diverse targets, which circumvent the use of established raw materials, presents a considerable data handling challenge that would be a good test of 
modern computer-assisted synthesis planning software (11-20). We enlisted the SYNTHIA ${ }^{T M}$ retrosynthesis platform $(12,16,20)$ to facilitate navigation of requisite parameters including availability, pricing, and novelty of starting materials, route brevity, issues of chemo-, regio-, and stereoselectivity, as well as route visualization, documentation, sharing and storage. We present herein predicted retrosynthetic routes to twelve diverse COVID-19 therapeutic candidates that initiate, as frequently as possible, with starting materials that are distinct from those used in published or patented syntheses. Few studies of modern retrosynthetic software validate routes experimentally, so it was important for us to realize some of our calculated routes.

\section{Results And Discussion}

Our study commenced with a crowd-sourcing approach wherein each member of our lab collected all published and patented synthetic routes for one of the drugs in Figure 1. The routes were then encoded via their simplified molecular-input line-entry system (SMILES) strings. From this dataset we built an interactive route visualizer, available for free at http://covidroutes.cernaklab.com (21), to facilitate review of existing routes. The concatenated list of starting material SMILES from each target was used as an exclusion criterion in each retrosynthetic search. This approach allowed us to rapidly navigate to novel starting materials. Each search result contained 50 route proposals, and the user-defined search heuristic was generally set to minimize starting material cost. A single search heuristic worked for most targets, but occasionally, the preference for minimized cost reagents would result in proposed routes with more reaction steps than desired. In these cases, the search heuristic was modified by relaxing the preference for reagent cost and increasing the software's beam search width. Predicted routes were manually reviewed for step count, synthetic feasibility, and ease of execution of proposed reactions on multikilogram scale, for instance by biasing towards routes that minimized the use of cryogenic cooling or pyrophoric reagents. The final heuristic used for each target is shown in the Supporting Information.

Among small molecules being considered (Figure 2), we focused on remdesivir (1), umifenovir (2), bromhexine (3), galidesivir (4), ritonavir (5), cobicistat (6), ribavirin (7), camostat (8), darunavir (9), nelfinavir (10), favipiravir (11), and baricitinib (12). In most cases the proposed route has the same number, or fewer, steps than the established routes, and initiates from distinct starting materials. Our analysis yielded alternate starting material proposals for 1-12, which can relieve pressure on the fine chemical supply chain. Using galidesivir (4) as an example (Figure 3), the software proposed a sequence hinging on a trans-hydroiodination of alkyne 13, an Evans alkylation to form 18, an Ullman coupling to form 19, and an enantioselective Heck-coupling to give 22. The software proposed that the latter reaction mixture could be subjected in situ to hydrochloric acid to remove the Boc-protecting groups in a one-pot operation. Dihydroxylation of $\mathbf{2 2}$ would complete the synthesis of 4. Exemplary starting materials 23-25 were excluded from the search based on the appearance of their SMILES strings in published routes to 4 . The algorithm successfully navigated around five established pyrrolopyrimidine starting materials to arrive at 21, which is cost-competitive with the established nucleobase sources; for instance, the 7-desbromo analog of 21,4 -chloro-5H-pyrrolo[3,2-d]pyrimidine, is listed at $\$ 9.90 / \mathrm{g}$ while a $4-\mathrm{OH}$ analog of 21 , 
7-bromo-5H-pyrrolo[3,2-d]pyrimidin-4-ol, which is used in the reported synthesis of 4, is listed at $\$ 280 / \mathrm{g}$ (22). On production scale, all starting material pricing would likely be customized based on competitive bidding, but in any event the high list price of $\mathbf{2 1}$ is comparable to starting material analogs currently described in patents. The proposed use of an Evans auxiliary to produce $\mathbf{1 8}$ highlights the software's desire to select robust chemistry, but this step could likely be replaced with a catalytic protocol to avoid auxiliary use for large-scale production if needed. Indeed, the overall route proposes a variety of catalytic operations. For the production of 1-12 on large scale, routes could be found that minimized use of cryogenic conditions, pyrophoric reagents or expensive catalysts, which were the main biases imposed in our manual review of answer sets beyond route length and starting material cost. For 1, novel starting materials were identified but the route bore high similarity to known routes, mirroring the automated retrosynthesis findings of the Gryzbowski lab for this target (10). While the software was challenged by esoteric functionalities like a chiral phosphorus atom, the predicted route to $\mathbf{4}$ discussed above, and those to $\mathbf{2}$ and $\mathbf{3}$ discussed below, represent typical outputs.

Umifenovir (2), is an antiviral drug developed to combat influenza infections whose use against SARSCoV1 made it an attractive synthetic target for this study. It is believed that $\mathbf{2}$ inhibits entry of viruses into human cells, and the antiviral has been used in many clinical trials as an investigational COVID-19 therapy (9). Although 2 shows promising in vitro activity against the novel coronavirus, recent clinical results suggest limited efficacy for 2 against COVID-19 in humans (23). Using the search criteria described above, we arrived at a series of routes to 2 based on the oxidative cyclization of an aniline with a b-ketoester (24). Since it is a commodity chemical, ethyl acetoacetate $\mathbf{2 7}$ was not included in the exclusion criteria of our heuristic and appears as a starting material here, although it has been used

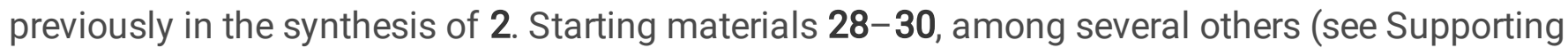
Information), were excluded. A key theme that separated the predicted routes from the established indoleforming routes, and enabled the use of distinct starting materials, was the incorporation of a BaeyerVilliger oxidation to utilize an acetyl group as a surrogate to the requisite hydroxyl group at $\mathrm{C} 1$. We found this proposal of a Baeyer-Villiger oxidation to be a surprising solution. Among other proposals that were non-obvious to us was the suggested $\mathrm{C}-\mathrm{H}$ oxidation of an ethyl group where the use of $\mathrm{C}-\mathrm{H}$ functionalization logic (25-28) reduces the cost of the starting materials. The software proposed an inefficient three-step sequence to $\mathrm{N}$-methylate the indole, whereas published syntheses of 2 reported $\mathrm{N}$ methylation directly from the indole with methyl iodide and sodium hydride. We opted to use this one-step precedent instead of the software's three-step proposal. In another search, a proposed sequence to 2 initiated with a pre-installed halogen coupling handle, instead of a $\mathrm{C}-\mathrm{H}$ bond, to enable a related indole formation, but instead invoked a different $\mathrm{C}-\mathrm{H}$ functionalization logic via a Bamberger rearrangement. As described below, these four routes were reduced to experimental practice with only minor modifications to reaction conditions and sequences proposed by the software.

To experimentally validate routes to $\mathbf{2}$ (Figure 4), we first investigated the proposed indole formation from 1-(4-aminophenyl)ethan-1-one $(26, \$ 1.15 / \mathrm{g})$ and ethyl acetoacetate $(27, \$ 0.03 / \mathrm{g})$ using oxidative reaction conditions (Figure 3, route A). Pretreatment of $\mathbf{2 6}$ and $\mathbf{2 7}$ with 1 mol\% indium(III) bromide, to form 31, was followed by oxidative cyclization to form 32 . While the published reaction conditions for the suggested 
reaction (24) did provide the desired indole 32 , the yield was only $20 \%$. Using magnesium sulfate to promote the formation of $\mathbf{3 1}$ improved the yield of $\mathbf{3 2}$ to $47 \%$. As described below, other implementations of this reaction gave much higher yields. $N$-Methylation occurred smoothly to produce 33 in $99 \%$ yield. An issue was encountered in the experimental realization of the Baeyer-Villiger oxidation using $m C P B A$ in that a mixture of oxidation products was obtained. Unstable products we believe to be from oxidation of the indole's double bond accounted the bulk of the reacted material, and only traces of $\mathbf{3 4}$ were isolated. While formation of $\mathbf{3 4}$ was accurately predicted, the subtle interplay of electronics that govern the preference for the desired Baeyer-Villiger oxidation over the undesired Prilezhaev oxidation could not be teased out by the software, and the best modification of reaction conditions we found yielded small amounts of $\mathbf{3 4}$ as a mixture with undesired oxidation products. A literature search on related indoles revealed that the a-chloroketone $\mathbf{3 5}$ should be a viable substrate for the Baeyer-Villiger (29), with the chloro-group acting as a directing group to favor oxidation of the ketone. We thus modified the route and, indeed, chlorination of $\mathbf{3} \mathbf{3}$ led to $\mathbf{3 5}$, which underwent selective Baeyer-Villiger oxidation to produce $\mathbf{3 6}$. Subsequent bromination produced $\mathbf{3 7}$, which underwent thioetherification with $\mathbf{3 8}$ and in situ saponification to produce 39. Here, the route intercepts known syntheses of 2 via alkylation with $\mathbf{4 0}$ (30). All intermediates predicted by the software were observed, but a modification to incorporate a chlorine directing group was necessary to achieve usable levels of selectivity in the formation of $\mathbf{3 6}$. This change led us to demonstrate the bromination of 36 to produce $\mathbf{3 7}$, instead of brominating $\mathbf{3 4}$, yet the selective bromination of $\mathbf{3 4}$ en route to $\mathbf{3 9}$ is a known reaction (31).

The output of a SYNTHIA ${ }^{\mathrm{TM}}$ search is a ranked list of route proposals. Several other computed routes to 2 were also experimentally vetted. One route, based on a variation of the same indole-formation and Baeyer-Villiger sequence described above, proposed a benzylic $\mathrm{C}-\mathrm{H}$ oxidation of indole $\mathbf{4 3}$ (Figure 4, route B). The indole-synthesis was more productive with $\mathbf{4 1}$ than with 26, yielding $\mathbf{4 2}$ in $\mathbf{7 9 \%}$ yield. Methylation gave $\mathbf{4 3}$ in $92 \%$ yield. SYNTHIA ${ }^{\text {TM }}$ predicts reaction sequences. Corresponding reaction conditions are recommended based on what was reported in the source literature. While these recommendations work well for a majority of substrates, exact recipes for specific substrates may require user direction. Software-recommended conditions of Oxone ${ }^{\circledR}$ with potassium bromide (32) for the $\mathrm{C}-\mathrm{H}$ oxidation of $\mathbf{4 3}$ were unsuccessful in our hands. An experimental survey of oxidants revealed the recently disclosed Baran-Roček oxidation $(33,34)$ could selectively oxidize $\mathrm{C} 14$ in $62 \%$ yield, thus intercepting the previous route to 2 . While the generation of chromium waste is only viable on small scale, this result validates the proposed $\mathrm{C}-\mathrm{H}$ functionalization hypothesis.

The direct installation of the chloromethyl ketone via a Friedel-Crafts acylation would provide a concise and alternative route to $\mathbf{2}$. Indeed, the software had proposed a route that used a Friedel-Crafts acylation (Figure 4, route $\mathrm{C}$ ). This route was intriguing in that it initiated the synthesis from $\mathbf{4 5}$, an exceptionally cheap starting material. While the software proposed a Friedel-Crafts acylation with acetyl chloride, we modified the route to use instead chloroacetyl chloride $(\mathbf{4 8}, \$ 0.13 / \mathrm{g})$, thus installing the chloride directing group in a single step. Experimentally, oxidative indole-coupling to form $\mathbf{4 6}$, followed by methylation to form 47, occurred smoothly. Friedel-Crafts acylation of $\mathbf{4 7}$ with $\mathbf{4 8}$ under influence of aluminum(III) 
chloride gave $\mathbf{3 5}$ and intercepted the other routes. The 2:1 regioselectivity of the acylation would require optimization for production on scale. Aside from this reaction, the regioselectivity for desired isomers was excellent for other $\mathrm{C}-\mathrm{H}$ functionalization events. While $\mathrm{C}-\mathrm{H}$ functionalization logic is not new, it has become rigorously adopted in synthesis only in recent years. We were therefore surprised that $\mathrm{C}-\mathrm{H}$ functionalization reactions were frequently proposed by the software, and expect this outcome is the result of the preference for low-cost starting materials in our heuristic, with $\mathrm{C}-\mathrm{H}$ bonds in many instances being cheaper than other functionalities. The Friedel-Crafts acylation route described functionalizes six $\mathrm{C}-\mathrm{H}$ bonds over seven reactions to convert $\mathbf{2 7}$ and $\mathbf{4 5}$ into 2.

We next employed a different tactic. Most routes to 2 hinge on a Nenitzescu indole coupling (30) between 1,4-benzoquinone and a b-aminocrotonic ester (21). Indeed, the Nenitzescu reaction using known starting materials featured as a proposal in our query results when default search criteria were used, so the keyword "Nenitzescu" was used as an exclusion criterion. This heuristic did not employ a SMILES exclusion criterion, so starting material $\mathbf{5 2}$ was employed even though this chemical has been used in a prior synthesis of $\mathbf{2}$. The results of this search led to yet another form of $\mathrm{C}-\mathrm{H}$ functionalization logic, via a Bamberger rearrangement to install the $\mathrm{C} 1$ hydroxyl (Figure 4, route D). SYNTHIA $\mathrm{A}^{\mathrm{TM}}$ proposed the use of 4-bromo-2-chloro-1-nitrobenzene as a starting material. In our hands, the requisite indole coupling on the chloride gave only traces of $\mathbf{3 9}$, and we ultimately modified the starting material to use 2,4-dibromo-1nitrobenzene (49) instead. This modification allowed the indole coupling to proceed, as discussed below, with the added benefit that $\mathbf{4 9}$ is cheaper than the corresponding chloride. In practice, $\mathbf{4 9}$ was reduced to the hydroxylamine, and treated with aqueous trifluoroacetic acid to affect the Bamberger rearrangement yielding $\mathbf{5 0}$, which was methylated to arrive at $\mathbf{5 1}$. Copper-catalyzed coupling to $\mathbf{5 3}$, itself obtained through the union of $\mathbf{5 2}$ and $\mathbf{3 8}$, produced $\mathbf{3 9}$ in $\mathbf{6 6 \%}$ yield when $\mathbf{5 4}$ was used as a ligand. These conditions were the result of a rapid optimization campaign using high-throughput experimentation (see Supporting Information). Subsequent alkylation of $\mathbf{3 9}$ with $\mathbf{4 0}$ produced $\mathbf{2}$. With this latter route, convergency is maximized, so the longest-linear sequence is just five steps.

Finally, we looked at 3 (Figure 5), a transmembrane protease, serine 2 (TMPRSS2) inhibitor that was being investigated in five clinical trials for COVID-19. A SYNTHIA ${ }^{\mathrm{TM}}$ search provided new reaction sequences of comparable length to known routes, identifying 55 as a novel starting material (21) by navigating around known starting materials 56-59 and others. The predicted route invoked a $\mathrm{C}-\mathrm{H}$ oxidation of the benzylic methyl group, presumably to arrive at cheaper starting materials, which readied 60 for a reductive amination with $\mathbf{6 1}$. The proposed route completed the synthesis of 3 by $N$-methylation of 62 with 63 . We considered instead that 3 could be synthesized from 2,4,6-tribromoaniline $(64, \$ 0.51 / \mathrm{g})$, which is used in the textile industry and readily available, with $N, N$-dimethylcyclohexylamine $(65$, $\$ 0.10 / \mathrm{g})$, a commodity chemical used in oil refining, via the direct $\mathrm{C}-\mathrm{H}$ functionalization recently reported by Shirakawa (35). While this manually designed route doesn't serve as a test of the software's capability per se, our motivation here was largely to do what we could as synthetic chemists to support production of a potentially beneficial medicine during a pandemic. The key reaction was added to the SYNTHIA ${ }^{T M}$ database so it would appear as a general solution to subsequent searches, and indeed this route came up 
as a top hit in a subsequent search for $\mathbf{3}$. To experimentally realize this one-step route, we found that 64 could be heated in excess $\mathbf{6 5}$ in the presence of tert-butylperoxide (35) to produce $\mathbf{3}$ in $41 \%$ yield. Further optimization of reaction conditions - to improve yield, ease of purification of $\mathbf{3}$, and address the hazard of using a peroxide on large scale - would be needed for commercial production (36). Nonetheless, this strategic disconnection reduces $\mathbf{3}$, in a single step, to starting materials that are considerably cheaper than those in commercial use.

Disconnection of drugs into affordable reagents was achieved for twelve drugs through the merger of crowd-sourcing and retrosynthetic software. Navigating the combinatorial explosion of routes towards twelve distinct synthetic targets to arrive at distinct and affordable starting materials was a data handling challenge that could only be navigated with automated retrosynthesis. Four predicted routes to 2 and one route to 3 , manually designed but added to the software's database for future use, were experimentally validated. Our work was performed over nine weeks in Spring 2020 against the backdrop of a developing pandemic. While full process development would require a longer timeline - for instance reagents such as peroxides would likely be replaced for production on scale - our results show that automated retrosynthetic predictions can rapidly de-risk the route scouting process of translational synthesis.

\section{Methods}

All reactions were conducted in oven- or flame-dried glassware under an atmosphere of nitrogen unless stated otherwise. Reactions were set up in an MBraun LABmaster Pro Glove Box $\left(\mathrm{H}_{2} \mathrm{O}\right.$ level $<0.1 \mathrm{ppm}, \mathrm{O}_{2}$ level $<0.1 \mathrm{ppm}$ ), or using standard Schlenk technique with a glass vacuum manifold connected to an inlet of dry nitrogen gas. Solvents (acetonitrile, tetrahydrofuran, dichloromethane) were purified using a MBraun SPS solvent purification system, by purging with nitrogen, and then passing the solvent through a column of activated alumina. Flash chromatography was performed on silica gel (230 - 400 Mesh, Grade 60) under a positive pressure of nitrogen. Thin Layer Chromatography was performed on $25 \mu \mathrm{m}$ TLC Silica gel $60 \mathrm{~F}_{254}$ glass plates purchased from Fisher Scientific (part number: S07876). Visualization was performed using ultraviolet light $(254 \mathrm{~nm})$, potassium permanganate $\left(\mathrm{KMnO}_{4}\right)$ stain, or Cerium Ammonium Molybdate (CAM) stain. See Supporting Information for additional details. Data used to produce the network visualizations in Figure 1, which are available as an interactive visualizer at http://covidroutes.cernaklab.com/, are also available in the Supporting Information.

\section{Declarations}

Acknowledgments The authors wish to thank Shane Krska and Melodie Christensen of Merck \& Co., Inc. for helpful discussions on reaction scalability. This work was supported by MilliporeSigma.

Author Contributions Y.L and Z.Z. performed synthetic chemistry experiments, B.M. developed the interactive route visualizer, Y.L., Z.Z., B.M., D.W., T.C., N.B. and S.T. performed SYNTHIA ${ }^{\mathrm{TM}}$ searches, Y.L., Z.Z., B.M., D.W., R.Z., E.S., A.M., Y.S., and T.C. collected and encoded literature and patent routes to 1-12, 
and contributed to writing the manuscript. Y.L., Z.Z., B.M., N.B., R.T., S.J., S.T. T.C. contributed to the study design. All authors reviewed and interpreted the data. T.C. supervised the work and wrote the manuscript.

Competing Interests Statement The Cernak Lab has received research funding or in-kind donations from MilliporeSigma, Relay Therapeutics, Janssen Therapeutics, SPT Labtech and Merck \& Co., Inc. T.C. holds equity in Scorpion Therapeutics, and is a co-Founder and equity holder of Entos, Inc.

\section{References}

1. "Temporary Policy for Preparation of Certain Alcohol-Based Hand Sanitizer Products During the Public Health Emergency (COVID-19) Guidance for Industry." (Food and Drug Administration, 2020). Available at https://www.fda.gov/media/136289/download.

2. L. Garbe, R. Rau, T. Toppe, Influence of perceived threat of Covid-19 and HEXACO personality traits on toilet paper stockpiling. PLOS ONE 15, e0234232 (2020).

3. H. Ledford, Dozens of coronavirus drugs are in development - what happens next? Nature 581, 247248 (2020).

4. R. K. Guy, R. S. DiPaola, F. Romanelli, R. E. Dutch, Rapid repurposing of drugs for COVID-19. Science 368, 829-830 (2020).

5. P. C. Fragkou, D. Belhadi, N. Peiffer-Smadja, C. D. Moschopoulos, F.-X. Lescure, H. Janocha, E. Karofylakis, Y. Yazdanpanah, F. Mentré, C. Skevaki, C. Laouénan, S. Tsiodras, Review of trials currently testing treatment and prevention of COVID-19. Clin. Microbiol. Infect. 26, 988-998 (2020).

6. COVID-19 Dashboard by the Center for Systems Science and Engineering (CSSE) at Johns Hopkins University (JHU). Available at https://coronavirus.jhu.edu/map.html (accessed December 9th, 2020)

7. M. A. Hardy, B. A. Wright, J. L. Bachman, T. B. Boit, H. M. S. Haley, R. R. Knapp, R. F. Lusi, T. Okada, V. Tona, N. K. Garg, R. Sarpong, Treating a Global Health Crisis with a Dose of Synthetic Chemistry. ACS Cent. Sci. 6, 1017-1030 (2020).

8. "Working to Supply Remdesivir for COVID-19." (I. Gilead Sciences, 2020). Available at https://www.gilead.com/purpose/advancing-global-health/covid-19/working-to-supply-remdesivirfor-covid-19.

9. D. Huang, H. Yu, T. Wang, H. Yang, R. Yao, Z. Liang. Efficacy and safety of umifenovir for coronavirus disease 2019 (COVID-19): A systematic review and meta-analysis. J. Med. Virol. 1-10 (2020).

10. S. Szymkuc, E. P. Gajewska, K. Molga, A. Wolos, R. Roszak, W. Beker, M. Moskal, P. Dittwald, B. A. Grzybowski, Computer-generated "synthetic contingency" plans at times of logistics and supply problems: scenarios for hydroxychloroquine and remdesivir. Chem. Sci. 11, 6736-6744 (2020).

11. E. J. Corey, W. T. Wipke, Computer-assisted design of complex organic syntheses. Science 166, 178192 (1969).

12. S. Szymkuc, E. P. Gajewska, T. Klucznik, K. Molga, P. Dittwald, M. Startek, M. Bajczyk, B. A. Grzybowski, Computer-Assisted Synthetic Planning: The End of the Beginning. Angew. Chem. Int. Ed. Engl. 55, 5904-5937 (2016). 
13. M. H. S. Segler, M. Preuss, M. P. Waller, Planning chemical syntheses with deep neural networks and symbolic Al. Nature 555, 604-610 (2018).

14. C. W. Coley, D. A. Thomas III, J. A. M. Lummiss, J. N. Jaworski, C. P. Breen, V. Schultz, T. Hart, J. S. Fishman, L. Rogers, H. Gao, R. W. Hicklin, P. P. Plehiers, J. Byington, J. S. Piotti, W. H. Green, A. J. Hart, T. F. Jamison, K. F. Jensen, A robotic platform for flow synthesis of organic compounds informed by Al planning. Science 365, eaax1566 (2019).

15. T. Badowski, E. P. Gajewska, K. Molga, B. A. Grzybowski, Synergy Between Expert and MachineLearning Approaches Allows for Improved Retrosynthetic Planning. Angew. Chem. Int. Ed. Engl. 59, 725-730 (2020).

16. T. Klucznik, B. Mikulak-Klucznik, M. P. McCormack, H. Lima, S. Szymkuc, M. Bhowmick, K. Molga, Y. Zhou, L. Rickershauser, E. P. Gajewska, A. Toutchkine, P. Dittwald, M. P. Startek, G. J. Kirkovits, R. Roszak, A. Adamski, B. Sieredzi-Ñska, M. Mrksich, S. L. J. Trice, B. A. Grzybowski, Effıcient Syntheses of Diverse, Medicinally Relevant Targets Planned by Computer and Executed in the Laboratory. Chem 4, 522-532 (2018).

17. K. Molga, P. Dittwald, B. A. Grzybowski, Navigating around Patented Routes by Preserving Specific Motifs along Computer-Planned Retrosynthetic Pathways. Chem 5, 460-473 (2019).

18. C. W. Coley, W. H. Green, K. F. Jensen, Machine Learning in Computer-Aided Synthesis Planning. Acc. Chem. Res. 51, 1281-1289 (2018).

19. A. A. Lee, Q. Yang, V. Sresht, P. Bolgar, X. Hou, J. L. Klug-McLeod, C. R. Butler, Molecular Transformer unifies reaction prediction, and retrosynthesis across pharma chemical space. Chem. Commun. 55, 12152-12155 (2019).

20. B. Mikulak-Klucznik, P. Gołębiowska, A. A. Bayly, O. Popik, T. Klucznik, S. Szymkuć, E. P. Gajewska, P. Dittwald, O. Staszewska-Krajewska, W. Beker, T. Badowski, K. A. Scheidt, K. Molga, J. Mlynarski, M. Mrksich, B. A. Grzybowski. Computational planning of the synthesis of complex natural products. Nature 588, 83-88 (2020).

21. Available at http://covidroutes.cernaklab.com.

22. Prices shown are the cheapest U.S. list price from a structure search on www.sigmaaldrich.com. For starting materials not available from this website, the price shown is the cheapest list price found in a SciFinder search of commercially available materials.

23. N. Lian, H. Xie, S. Lin, J. Huang, J. Zhao, Q. Lin. Umifenovir treatment is not associated with improved outcomes in patients with coronavirus disease 2019: a retrospective study. Clin. Microbiol. Infect. 26, 917-921 (2020).

24. S. Würtz, S. Rakshit, J. J. Neumann, T. Dröge, F. Glorius, Palladium-Catalyzed Oxidative Cyclization of N-Aryl Enamines: From Anilines to Indoles. Angew. Chem. Int. Ed. Engl. 47, 7230-7233 (2008).

25. T. Newhouse, P. S. Baran, If C-H Bonds Could Talk: Selective C-H Bond Oxidation. Angew. Chem. Int. Ed. Engl. 50, 3362-3374 (2011).

26. T. Cernak, K. D. Dykstra, S. Tyagarajan, P. Vachal, S. W. Krska, The medicinal chemist's toolbox for late stage functionalization of drug-like molecules. Chem. Soc. Rev. 45, 546- 576 (2016). 
27. H. M. L. Davies, D. Morton, Collective Approach to Advancing C-H Functionalization. ACS Cent. Sci. 3, 936-943 (2017).

28. E. J. Corey, The logic of chemical synthesis (Nobel foundation, [Nobelstiftelsen], 1991).

29. S. Nakatsuka, O. Asano, K. Ueda, T. Goto, Introduction of a hydroxy group onto 5- and 6-position of indole nucleus by Friedel-Crafts acylation and subsequent Baeyer-Villiger Oxidation. Heterocycles 26, 1471-1474 (1987).

30. K. V. Balakin, R. Filosa, S. N. Lavrenov, A. S. Mkrtchyan, M. B. Nawrozkij, I. A. Novakov, Arbidol: a quarter-century after. Past, present and future of the original Russian antiviral. Russ. Chem. Rev. 87, 509-552 (2018).

31. Z. V. F. Wright, N. C. Wu, R. U. Kadam, I. A. Wilson, D. W. Wolan, Structure-based optimization and synthesis of antiviral drug Arbidol analogues with significantly improved affinity to influenza hemagglutinin. Bioorg. \& Med. Chem. 27, 3744-3748 (2017).

32. K. Moriyama, M. Takemura, H. Togo, Direct and Selective Benzylic Oxidation of Alkylarenes via C-H Abstraction Using Alkali Metal Bromides. Org. Lett. 14, 2414-2417 (2012).

33. Y. Kanda, H. Nakamura, S. Umemiya, R. K. Puthukanoori, V. R. M. Appala, G. K. Gaddamanugu, B. R. Paraselli, P. S. Baran, Two-Phase Synthesis of Taxol. J. Am. Chem. Soc. 142, 10526-10533 (2020).

34. M. Krumpolc, J. Roček, Synthesis of stable chromium(V) complexes of tertiary hydroxy acids. J. Am. Chem. Soc. 101, 3206-3209 (1979).

35. R. Ueno, Y. Ikeda, E. Shirakawa, tert-Butoxy-Radical-Promoted a-Arylation of Alkylamines with Aryl Halides. Eur. J. Org. Chem. 2017, 4188-4193 (2017).

36. Care must be taken in optimization studies based on peroxide activation, as we observed a violent reaction on a small-scale between neat 65 and benzoylperoxide.

\section{Figures}




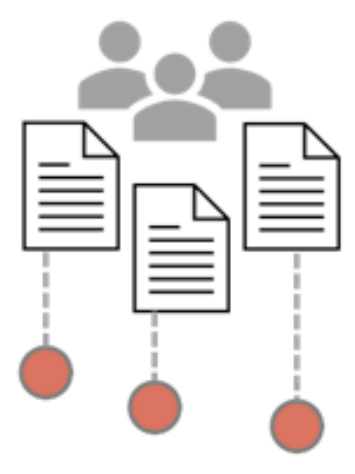

step 1.

Crowd-sourced literature review identifies known starting materials

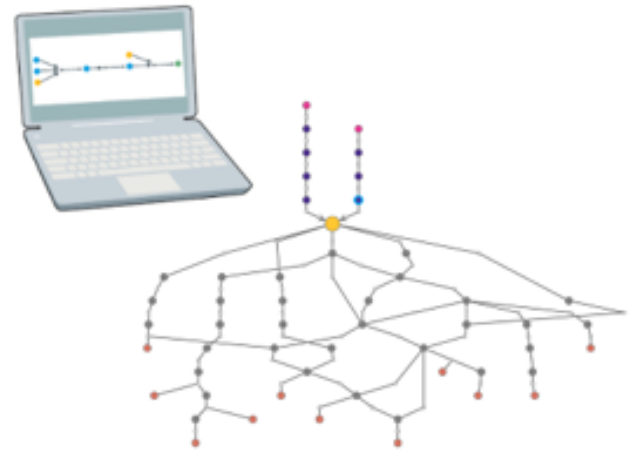

step 2.

Retrosynthetic search specifically excludes known starting materials

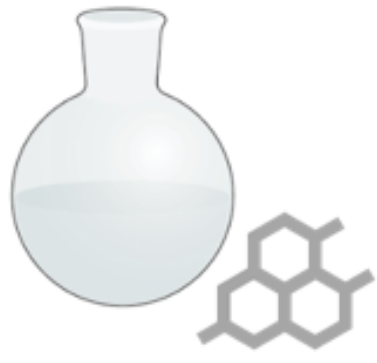

step 3.

Experimental validation of synthetic route

Figure 1

Workflow to identify alternate starting material supply chains. 

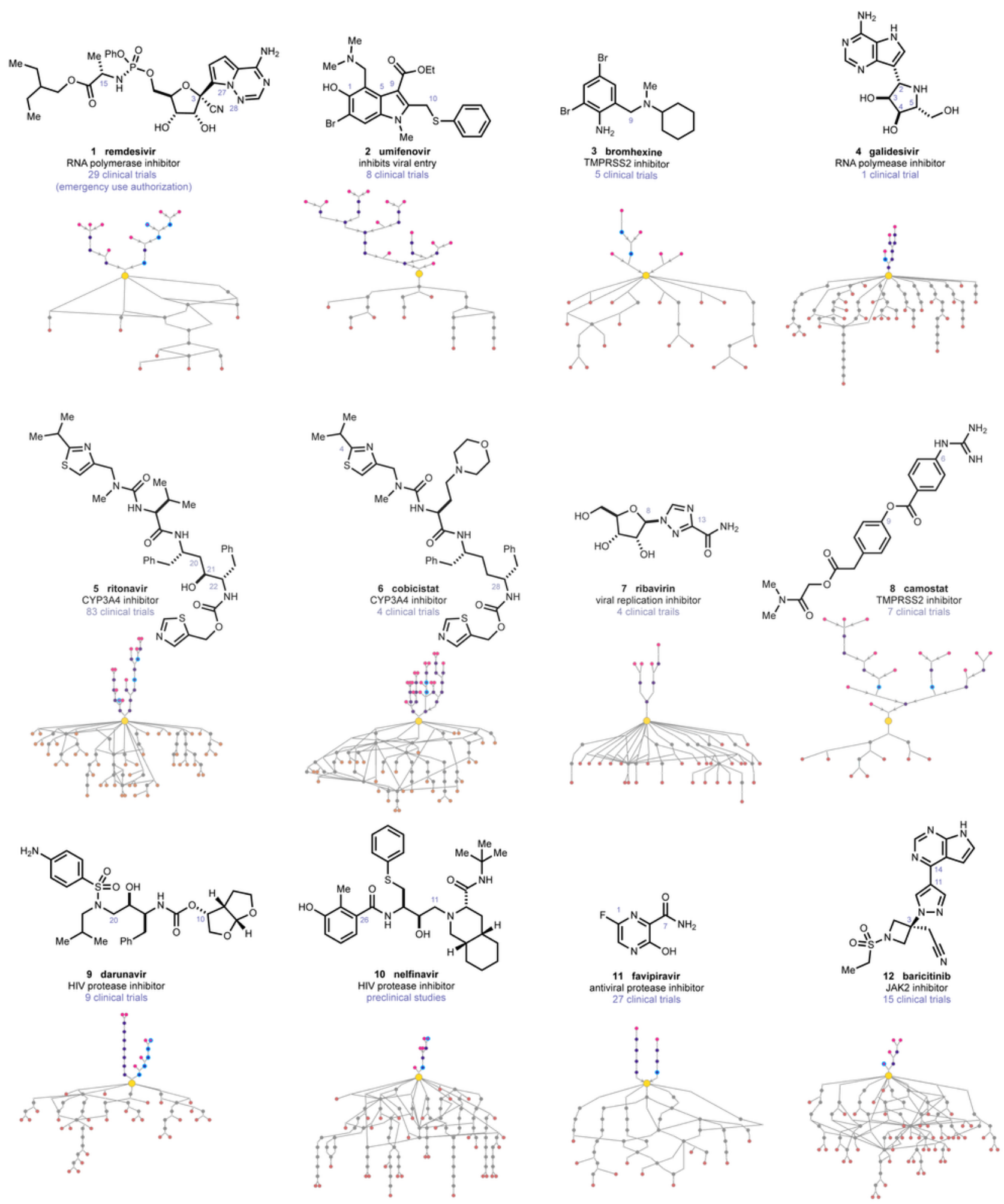

\section{Figure 2}

Therapeutics being evaluated for COVID-19 and their retrosynthetic analysis networks. The number of clinical trials is based on search results for all listed trials, completed, active, or planned, found on www.clinicaltrials.gov (accessed July 7th, 2020). For the route networks, the yellow dot at center is the target molecule. Routes in grey and orange below the target are published, and the routes in purple and pink above the target are routes predicted. Intersection nodes in literature routes reflect common 
intermediates or starting materials. An interactive version of this diagram is available as an interactive route visualizer.
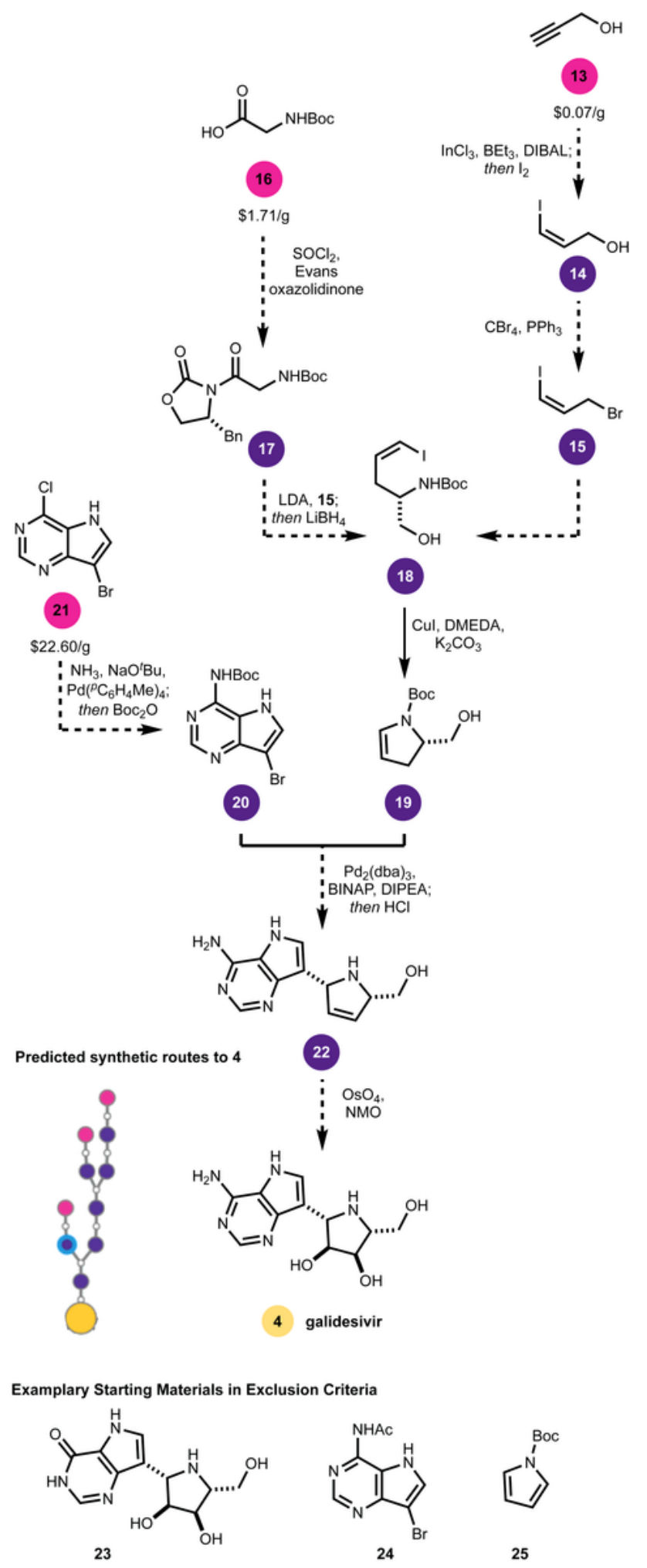

Figure 3

Predicted synthetic sequence to 4 . Reaction conditions are proposals from the SYNTHIA ${ }^{\mathrm{TM}}$ software. 


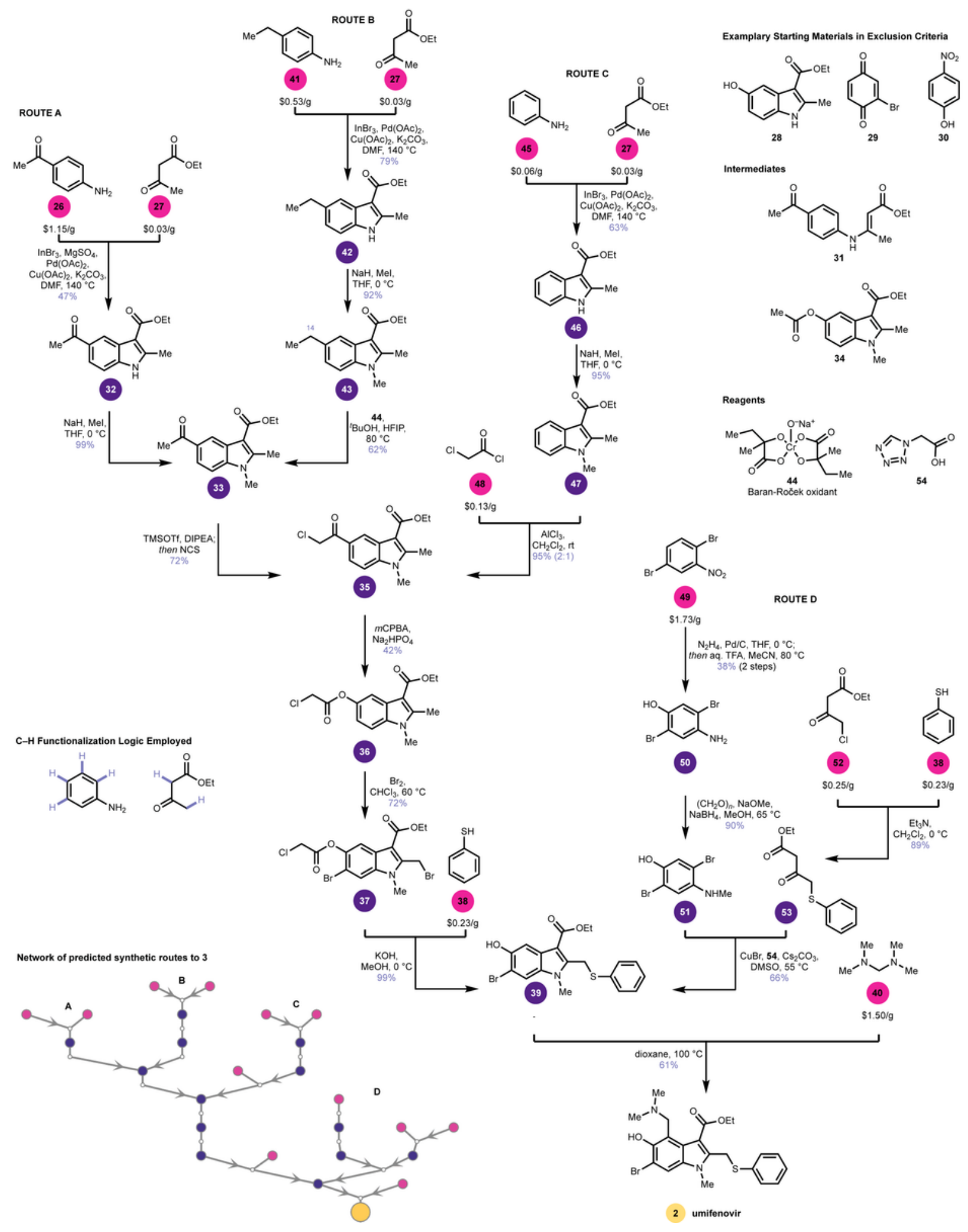

Figure 4

Experimental demonstration of four routes to 2. Each route terminates in inexpensive starting materials. $26,41,45,48$ and 49 have not been used in a published synthesis of 2 . The conversion of 33 to 35 , and the use of starting material 49, instead of 4-bromo-2-chloro-1-nitrobenzene, were manual modifications to the software's prediction. See main text for details. 


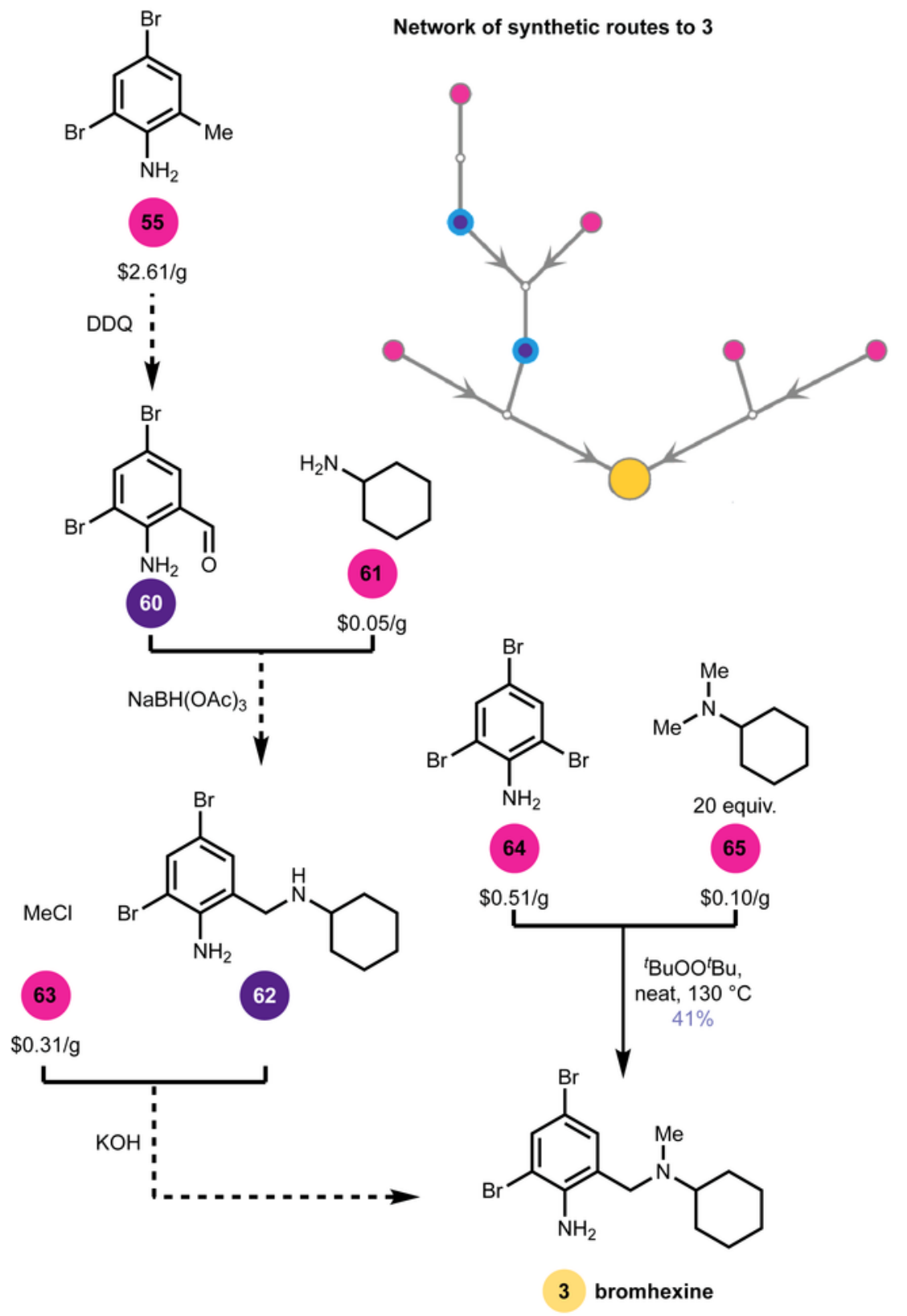

Examplary Starting Materials in Exclusion Criteria<smiles>COC(=O)c1ccccc1N</smiles>

56<smiles>Cc1ccccc1[N+](=O)[O-]</smiles>

57<smiles>O=[N+]([O-])c1ccccc1CBr</smiles>

58<smiles>CNC1CCCCC1</smiles>

59

\section{Figure 5}

Predicted and realized routes to 3. Dashed arrows are predicted, while the solid arrow is manually designed and experimentally validated, with subsequent addition of the key reaction to the SYNTHIA ${ }^{\text {TM }}$ database.

\section{Supplementary Files}


This is a list of supplementary files associated with this preprint. Click to download.

- SynthiaCOVIDSI081621.docx 